\title{
On Some Configurations Related to the Shelah Weak Hypothesis
}

\author{
Moti Gitik ${ }^{1}$ and Saharon Shelah ${ }^{2}$ \\ ${ }^{1}$ School of Mathematical Sciences \\ Tel Aviv University \\ Tel Aviv 69978, Israel \\ ${ }^{2}$ Department of Mathematics \\ Hebrew University of Jerusalem \\ Jerusalem, Israel
}

\begin{abstract}
We show that some cardinal arithmetic configurations related to the negation of the Shelah Weak Hypothesis and natural from the forcing point of view are impossible.
\end{abstract}

\section{Introduction}

The Shelah Weak Hypothesis (SWH) formulated in [Sh:400A] states that for every cardinal $\lambda$ the number of singular cardinals $\kappa<\lambda$ with $p p \kappa \geq \lambda$ is at most countable. The negation of SWH is one of the weakest statements on cardinal arithmetic whose consistency is unknown. Clearly, SWH follows from GCH or even from the Shelah Strong Hypothesis saying that for every singular $\kappa, p p \kappa=\kappa^{+}$. On the other hand by [Sh-g], " $|p c f a|>|a|$ " implies $\neg$ SWH. The forcing construction of [Gi-Sh] and [Gi-Ma] show that any finite or countable number of $\kappa$ 's with $p p \kappa>\lambda$ is consistently possible.

The present paper grew from an attempt made by the first author to force $\neg \mathrm{SWH}$ using a forcing of type of [Gi]. One of the features of this forcing is that it does not add new bounded subsets to a cardinal while blowing its power. Here we show (in ZFC) that some configurations which are very natural from the forcing point of view are just impossible.

The first theorem under stronger assumptions was proved by the first author; the second author was able to weaken the assumptions and find a more elegant proof. Most of the 
generalizations are due to the second author. The second theorem is due solely to the second author.

\section{Main Results}

Theorem 1 The following is impossible:

(a) $\kappa_{1}<\kappa_{*}, c f \kappa_{1}=\aleph_{0}, c f \kappa_{*}>2^{\aleph_{0}}$

(b) for every large enough $\mu<\kappa_{1}$ of cofinality $\left(2^{\aleph_{0}}\right)^{+}$we have $p p \mu=\mu^{+}$

(c) $\kappa_{*}=\sup \left\{\mu \mid \mu<\kappa_{*}, c f \mu=\aleph_{0}\right.$ and $\left.p p \mu>\kappa_{*}^{+}\right\}$

(d) there are a strictly increasing sequence $\left\langle\lambda_{\alpha} \mid \alpha<c f \kappa_{*}\right\rangle$ of regular cardinals between $\kappa_{1}$ and $\kappa_{*}$ unbounded in $\kappa_{*}$, a filter $D$ on $\omega$ containing all cofinite subsets of $\omega$ and a sequence of functions $\left\langle f_{\lambda_{\alpha}} \mid \alpha<c f \kappa_{*}\right\rangle$ such that

( $\alpha) f_{\lambda_{\alpha}}: \omega \rightarrow \operatorname{Reg} \cap \kappa_{1} \backslash\left(2^{\aleph_{0}}\right)^{+}$

( $\beta) \lim _{D} f_{\lambda_{\alpha}}=\kappa_{1}$

$(\gamma) \lambda_{\alpha}=\operatorname{tcf}\left(\prod_{n<\omega} f_{\lambda_{\alpha}}(n) / D\right)$

$(\delta) \alpha<\beta<c f \kappa_{*}$ implies $f_{\lambda_{\alpha}}<_{D} f_{\lambda_{\beta}}$

$(\epsilon)$ if $\alpha<\beta<c f \kappa_{*}$ and $\lambda \in \operatorname{Reg} \cap \lambda_{\beta} \backslash \lambda_{\alpha}^{+}$then there is a function $f_{\lambda}: \omega \rightarrow$ Reg $\cap \kappa_{1} \backslash\left(2^{\aleph_{0}}\right)^{+}$such that $f_{\lambda_{\alpha}}<_{D} f_{\lambda}<_{D} f_{\lambda_{\beta}}$ and $\lambda=\operatorname{tcf}\left(\prod_{n<\omega} f_{\lambda}(n) / D\right)$.

\section{Discussion 1.1}

(1) The assumption (c) is a form of $\neg \mathrm{SWH}$ which claims that there are more than $2^{\aleph_{0}}$ singular cardinals of cofinality $\aleph_{0}$ with $p p$ above their supremum.

(2) The assumption (d) holds naturally in forcing constructions with $D=$ the filter of cofinite subsets of $\omega$. But it seems to be problematic in ZFC. In [Sh-g, II§1] proof of a weak relative is a major result.

(3) See $[$ Sh-g, VI] for uncountable cofinalities.

Proof. Suppose otherwise. W. 1. of g. we can assume that $c f \kappa_{*}=\left(2^{\aleph_{0}}\right)^{+}$. Also, replacing $\left\langle\lambda_{\alpha} \mid \alpha<\left(2^{\aleph_{0}}\right)^{+}\right\rangle$by its restriction to an unbounded subset, we can assume that the following holds (see [Sh-g] for this type of argument) 
$(*)$ for every $n<\omega,\left\langle f_{\lambda_{\alpha}}(n) \mid \alpha<\left(2^{\aleph_{0}}\right)^{+}\right\rangle$is strictly increasing and, if $f_{*}(n)=\bigcup_{\alpha<\left(2^{\aleph_{0}}\right)^{+}} f_{\lambda_{\alpha}}(n)$ then $f_{*}(n)<f_{\lambda_{0}}(n+1)$.

Now for every $\alpha<\left(2^{\aleph_{0}}\right)^{+}$and $\lambda \in \operatorname{Reg} \cap \lambda_{\alpha+1} \backslash \lambda_{\alpha}$ we use $(\epsilon)$ and find a function $f_{\lambda}: \omega \rightarrow \operatorname{Reg} \cap \kappa_{1} \backslash\left(2^{\aleph_{0}}\right)^{+}$such that $\lambda=\operatorname{tcf}\left(\prod_{n<\omega} f_{\lambda}(n) / D\right)$ and for every $n<\omega, f_{\lambda_{\alpha}}(n)<$ $f_{\lambda}(n)<f_{\lambda_{\alpha+1}}(n)$.

Clearly, $\left\langle f_{*}(n) \mid n<\omega\right\rangle$ is strictly increasing with limit $\kappa_{1}$ and $\operatorname{cf}\left(f_{*}(n)\right)=\left(2^{\aleph_{0}}\right)^{+}$ for every $n<\omega$. Using (b), we can assume removing finitely many $n$ 's, if necessary, that $p p\left(f_{*}(n)\right)=\left(f_{*}(n)\right)^{+}$for every $n<\omega$. Let $D_{*}$ by an ultrafilter on $\omega$ extending $D$. Let $\mu_{*}=t c f \prod_{n<\omega}\left(\left(f_{*}(n)\right)^{+} / D_{*}\right)$. It is well defined since $D_{*}$ is an ultrafilter. By (c), w.l. of g., for every $\alpha<\left(2^{\aleph_{0}}\right)^{+}$there is $\kappa_{\alpha}, \lambda_{\alpha}<\kappa_{\alpha}<\lambda_{\alpha+1}, c f \kappa_{\alpha}=\aleph_{0}$ and $p p \kappa_{\alpha} \geq \kappa_{*}^{++}$. Hence, there are $\tau_{\alpha, n}^{2} \in \operatorname{Reg} \cap \kappa_{\alpha} \backslash \lambda_{\alpha}^{++}(n<\omega)$ and a filter $D_{\alpha}$ on $\omega$ continuing all cofinite subsets of $\omega$ such that $\kappa_{*}^{++}=t c f\left(\prod_{n<\omega} \tau_{\alpha, n}^{2} / D_{\alpha}\right)$. By [Sh-g], we can then find $\tau_{\alpha, n}^{1} \in \operatorname{Reg} \cap \tau_{\alpha, n}^{2} \backslash \lambda_{\alpha}^{+}$such that $\kappa_{*}^{+}=t c f\left(\prod_{n<\omega} \tau_{\alpha, n}^{1} / D_{\alpha}\right)$ (note that we are doing this separately for each $\left.\alpha<\left(2^{\aleph_{0}}\right)^{+}\right)$. Let $a_{\alpha}^{m, \ell}=\left\{f_{\tau_{\alpha, n}}(m) \mid n<\omega\right\}$ for every $m<\omega$ and $\ell \in\{1,2\}$. Set $a_{\alpha}^{m}=a_{\alpha}^{m, 1} \cup a_{\alpha}^{m, 2}, a^{m}=$ $\bigcup_{\alpha<\left(2^{\left.\aleph_{0}\right)^{+}}\right.} a_{\alpha}^{m}$ and $a=\bigcup_{m<\omega} a^{m}$. All these sets consists of regular cardinals above $\left(2^{\aleph_{0}}\right)^{+}, a_{\alpha}^{m}$ 's are countable, $a^{m}$ 's and $a$ have cardinality of at most $\left(2^{\aleph_{0}}\right)^{+}$. Also $a_{\alpha}^{m} \subseteq\left[f_{\lambda_{\alpha}}(m), f_{\lambda_{\alpha}}(m+1)\right)$. Clearly, $a^{m}(m<\omega)$ is an unbounded subset of $f_{*}(m) \cap R e g$ of order type $\left(2^{\aleph_{0}}\right)^{+}$, since $\left\langle f_{\lambda_{\alpha}}(m) \mid \alpha<\left(2^{\aleph_{0}}\right)^{+}\right\rangle$is increasing with limit $f_{*}(m)$. Then, $\left(f_{*}(m)\right)^{+}=t c f\left(\prod a^{m} / J_{a^{m}}^{\text {bounded }}\right)$, as $p p\left(f_{*}(m)\right)=\left(f_{*}(m)\right)^{+}$. Hence, by $\left[\right.$Sh-g], $\left(f_{*}(m)\right)^{+} \in p c f a^{m} \subseteq p c f a$, for every $m<\omega$. Again, by [Sh-g], $p c f\left(\left\{\left(f_{*}(m)\right)^{+} \mid m<\omega\right\}\right) \subseteq p c f a$. But $\mu_{*}=\operatorname{tcf}\left(\prod_{n<\omega}\left(f_{*}(n)\right)^{+} / D_{*}\right)$, hence $\mu_{*} \in p c f a$. Let $\left\langle b_{\sigma} \mid \sigma \in p c f a\right\rangle$ be a generating sequence for $a$ (see [Sh-g] or [Sh:506]). W.l. of g. if $\mu_{*} \neq \kappa_{*}^{+\ell}$ for $\ell \in\{1,2\}$, then $b_{\mu_{*}} \cap b_{\kappa_{*}^{+\ell}}=\emptyset$. Let $\ell^{*} \in\{1,2\}$ be such that $\mu_{*} \neq \kappa_{*}^{+\ell^{*}}$.

Claim 1.2 The set $A=\left\{m<\omega \mid\right.$ for some $\left.\alpha<\left(2^{\aleph_{0}}\right)^{+}, \bigcup_{\beta \in\left[\alpha,\left(2^{\aleph_{0}}\right)^{+}\right)} a_{\beta}^{m} \subseteq b_{\mu_{*}}\right\}$ is in $D_{*}$.

Proof. Otherwise $\omega \backslash A \in D_{*}$ and for $m \in \omega \backslash A, f_{*}(m)=\sup \left(a^{m} \backslash b_{\mu_{*}}\right)$. Hence $\left(f_{*}(m)\right)^{+} \in$ $p c f\left(a \backslash b_{\mu_{*}}\right)$. So, $p c f\left(\left\{\left(f_{*}(m)\right)^{+} \mid m \in \omega \backslash A\right\}\right) \subseteq p c f\left(a \backslash b_{\mu_{*}}\right)$. But $\omega \backslash A \in D_{*}$ and $\mu_{*}=$ $t c f\left(\prod_{m<\omega}\left(f_{*}(m)\right)^{+} / D_{*}\right)$. Hence $\mu_{*} \in p c f\left(a \backslash b_{\mu_{*}}\right)$. Contradicting the choice of $b_{\mu_{*}}$.

$\square$ of the claim.

For $m \in A$ let $\alpha_{m}$ be the minimal $\alpha$ such that $\bigcup_{\beta \in\left[\alpha,\left(2^{\left.\left.\alpha_{0}\right)^{+}\right)}\right.\right.} a_{\beta}^{m} \subseteq b_{\mu_{*}}$. Set $\alpha_{*}=\bigcup_{m \in A} \alpha_{m}$. Clearly, $\alpha_{*}<\left(2^{\aleph_{0}}\right)^{+}$. Let $a^{\prime}=\bigcup\left\{a_{\beta}^{m} \mid m \in A, \beta \in\left[\alpha_{*},\left(2^{\aleph_{0}}\right)^{+}\right)\right\}$. Then $a^{\prime} \subseteq b_{\mu_{*}}$ and hence $\kappa_{*}^{+\ell^{*}} \notin p c f a^{\prime}$. However, $m \in A$ and $n<\omega$ imply that $f_{\tau_{\alpha_{*}, n}^{* *}}(m) \in a_{\alpha_{*}}^{m \ell^{*}} \subseteq a_{\alpha_{*}}^{m} \subseteq a^{\prime}$. So, for each $n<\omega$ we have

$$
\left\{f_{\tau_{\alpha *, n}^{*}}(m) \mid m \in A\right\} \subseteq a^{\prime} .
$$

Hence $p c f\left\{f_{\tau_{\alpha_{*}^{*}, n}^{*}}(m) \mid m \in A\right\} \subseteq p c f a^{\prime}$. But as $A \in D_{*}, \tau_{\alpha_{*}, n}^{\ell^{*}}=t c f\left(\prod_{m \in A} f_{\tau_{\alpha *, n}^{\ell^{*}}}(m) / D\right)$. So, for every $n<\omega, \tau_{\alpha_{*}, n}^{\ell^{*}} \in p c f a^{\prime}$. Then by [Sh-g], pcf $\left\{\tau_{\alpha^{*}, n}^{\ell^{*}} \mid n<\omega\right\} \subseteq p c f a^{\prime}$. But $\kappa_{*}^{+\ell^{*}}=t c f\left(\prod_{n<\omega} \tau_{\alpha^{*}, n}^{\ell^{*}} / D_{\alpha^{*}}\right)$. So, $\kappa_{*}^{+\ell^{*}} \in p c f a^{\prime}$. Contradiction. 


\section{Remark 1.3}

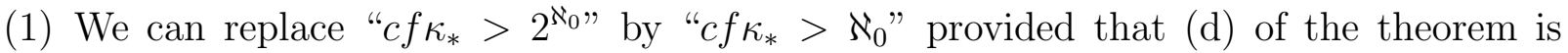
strengthened by adding the condition $(*)$ introduced in the beginning of the proof and $\left(2^{\aleph}\right)^{+}$is replaced by $\aleph_{1}$ in $(\mathrm{b})$.

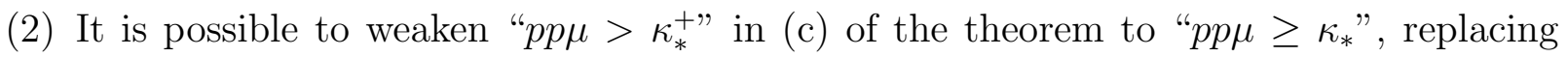
$\left(2^{\aleph_{0}}\right)^{+}$in $(\mathrm{b})$ by $\aleph_{1}$. Just after $(*)$ is obtained using $c f \kappa_{*} \geq\left(2^{\aleph_{0}}\right)^{+}$, we can replace $\kappa_{*}, \kappa_{*}^{+}, \kappa_{*}^{++}$by the limit of first $\aleph_{1}, \lambda_{\alpha}$ 's, its successor and its double successor. This is provided that for every $\alpha<\omega_{1}$ there is $\kappa_{\alpha}, \lambda_{\alpha}<\kappa_{\alpha}<\lambda_{\alpha+1}$ with $p p \kappa_{\alpha} \geq \lambda_{*}^{++}$, where $\lambda_{*}=\bigcup_{\alpha<\omega_{1}} \lambda_{\alpha}$. The condition " $p p \mu \geq \kappa_{*}$ " can be used easily to construct such $\left\langle\lambda_{\alpha} \mid \alpha<\omega_{1}\right\rangle$.

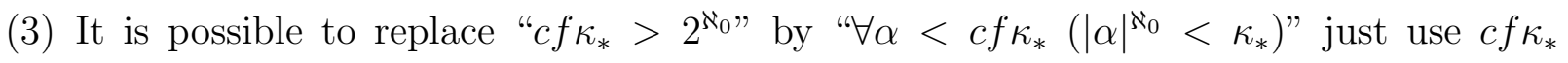
instead of $\left(2^{\aleph_{0}}\right)+$ in the proof.

The following is parallel to Solovay's result that SCH holds above a strongly compact cardinal.

Corollary 1.4 Suppose that the following holds: $\kappa$ is a cardinal such that

(a) for any given cardinal $\lambda$ it is possible to force $2^{\kappa} \geq \lambda$ by $\kappa^{++}$-c.c. forcing not adding new bounded subsets to $\kappa$ and adding $\lambda \omega$-sequences $\left\langle f_{\alpha} \mid \alpha<\lambda\right\rangle$ to $\kappa$ such that $\alpha<\beta \rightarrow f_{\alpha}<f_{\beta}$ (mod finite) and $\delta \in(\kappa, \lambda]$ regular cardinal implies that $f_{\delta}(n)$ is regular cardinal for every $n<\omega$.

(b) $p p(\mu)=\mu^{+}$for every large enough $\mu<\kappa$ of cofinality $\aleph_{1}$.

Then above $\kappa$ the following version of $S W H$ holds:

for every cardinal $\lambda$ the set $\left\{\mu \mid \kappa<\mu<\lambda\right.$, cf $\left.\mu=\aleph_{0}, p p \mu>\lambda^{+}\right\}$is at most countable.

Remark. Forcing notion of [Gi-Ma] or [Gi] satisfy (a).

Proof. Suppose otherwise. Let $\kappa_{*}$ be the first cardinal such that the set $\{\mu \mid \kappa<\mu<$ $\left.\kappa_{*}, c f \mu=\aleph_{0}, p p \mu>\kappa_{*}^{+}\right\}$is uncountable. Clearly, $c f \kappa_{*}=\aleph_{1}$. Now we force with the forcing of (a) and make $2^{\kappa} \geq \kappa_{*}$. The $\omega$-sequences produced by such forcing will satisfy $(*)$ of the proof of Theorem 1 with $D$ equal to the filter of cofinite sets. The chain condition of the forcing insures that the cardinal arithmetic does not change above $\kappa$. No new bounded subsets are added to $\kappa$, hence (b) of the statement of the corollary still holds. Now Theorem 
1 (actually using 1.3(2)) provides a contradiction.

Repeating the proof of Theorem 1 we can show the following generalization:

Theorem 1.5 The following is impossible:

(a) $\kappa_{1}<\kappa_{*}, c f \kappa_{1}=\aleph_{0}, c f \kappa_{*}>2^{\aleph_{0}}$

(b) there is $\ell, 1 \leq \ell<\omega$ such that for every $\mu<\kappa_{1}$ of cofinality $\left(2^{\aleph_{0}}\right)^{+}$we have $p p \mu \leq \mu^{+\ell}$

(c) $\kappa_{*}=\sup \left\{\mu \mid \mu<\kappa_{*}, c f \mu=\aleph_{0}\right.$ and $\left.p p \mu>\kappa_{*}^{+\ell}\right\}$

(d) the same as in Theorem 1.

If we allow infinite gaps between $\mu$ and $p p \mu$ in (b) of 1.5, then the following can be shown:

Theorem 1.6 Assume that

(a) $\kappa_{1}<\kappa_{*}, c f \kappa_{1}=\aleph_{0}, c f \kappa_{*}=\theta>\aleph_{0}, \alpha^{*}<\kappa_{1}, c f \alpha^{*}>\aleph_{0}$

(b) for every large enough $\mu<\kappa_{1}$ of cofinality $\theta$ we have $p p(\mu)<\mu^{+\alpha^{*}}$

(c) for some $\beta^{*}, \kappa_{*}=\sup \left\{\mu \mid \mu<\kappa_{*}, c f \mu=\aleph_{0}\right.$ and $\left.p p \mu \geq \kappa_{*}^{+\beta^{*}}\right\}$

(d) the condition (d) of Theorem 1 and $(*)$ of its proof

Then $\beta^{*}<\sigma^{+4}$ for some $\sigma<\alpha^{*}$.

Sketch of the proof. Suppose otherwise. We define $f_{*}(n)$ 's as in Theorem 1 . Now $c f f_{*}(n)=\theta$ and so $p p\left(f_{*}(n)\right)<\left(f_{*}(n)\right)^{+\alpha^{*}}$ for every $n<\omega$. Find $\sigma<\alpha^{*}$ such that for every $n<\omega, p p\left(f^{*}(n)\right) \leq\left(f_{*}(n)\right)^{+\sigma}$. Here we use that $c f \alpha^{*}>\aleph_{0}$. Instead of one $\mu_{*}$ in the proof of Theorem 1 (or finitely many cardinals in 1.6) we consider $p c f\left\{\left(f_{*}(n)\right)^{+\sigma^{\prime}} \mid n<\right.$ $\left.\omega, \sigma^{\prime} \leq \sigma\right\} \cap\left(\kappa_{*}, \kappa_{*}^{+\beta^{*}}\right]$. By the assumption we made, $\beta^{*} \geq \sigma^{+4}$. Then there should be $\kappa_{*}^{+\ell^{*}} \notin p c f\left\{\left(f_{*}(n)\right)^{+\sigma^{\prime}} \mid n<\omega, \sigma^{\prime} \leq \sigma\right\}$ for some $\ell^{*}, 1 \leq \ell^{*} \leq \beta^{*}$. This follows by results of [Sh:g, IX], see also [Sh:g, E12, 4.18 (b)]. The rest of the proof is as those of Theorem 1, only we use [Sh:g, I, 3.2(5)] to include $p c f\left\{\left(f_{*}(n)\right)^{+\sigma^{\prime}} \mid n<\omega, \sigma^{\prime} \leq \sigma\right\}$ into a union of finitely many $p c f$-generators.

Now we turn to another theorem which provides a different proof of Theorem 1 and some of its generalizations.

Theorem 2 Suppose that 
(a) $\kappa_{0}<\kappa_{1}<\kappa_{*}, 1 \leq n^{*}<\omega, n^{*}<\gamma^{*}<\theta$ and $\gamma^{*}$ is a successor ordinal

(b) $\theta=c f d \theta<\kappa_{0}$ and for every $\alpha<\theta,|\alpha|^{\aleph_{0}}<\theta$

(c) $c f \kappa_{1}=\aleph_{0}$ and $p p\left(\kappa_{1}\right) \geq \kappa_{*}^{+\gamma^{*}}$

(d) if $\mu \in\left(\kappa_{0}, \kappa_{1}\right)$ and $c f \mu=\theta$ then $p p \mu \leq \mu^{+n^{*}}$.

Then the following holds

(1) For every nonprincipal ultrafilter $D$ on $\omega$ and a sequence $\bar{\sigma}^{*}=\left\langle\sigma_{\ell}^{*} \mid \ell<\omega\right\rangle$ with $\kappa_{1}=\lim _{D} \bar{\sigma}^{*}$ and $\sigma_{\ell}^{*}(\ell<\omega)$ a limit cardinal of cofinality $\geq \theta$ in the interval $\left(\kappa_{0}, \kappa_{1}\right)$ there are a set $w \subseteq \gamma^{*}+1$ consisting of at most $n^{*}$ elements and a sequence $\bar{\sigma}^{* *}=$ $\left\langle\sigma_{\ell}^{* *} \mid \ell<\omega\right\rangle, \kappa_{0}<\sigma_{\ell}^{* *}<\sigma_{\ell}^{*}(\ell<\omega)$ such that

$(*)_{1}$ if $a \in\left[R_{D, \bar{\sigma}^{*}, \bar{\sigma}^{* *}}\right]^{\aleph_{0}}, \beta \leq \gamma^{*}$ and $\kappa_{*}^{+\beta} \in \operatorname{pcfa}$ then $\beta \in w$, where $R_{D, \bar{\sigma}^{*}, \bar{\sigma}^{* *}}=\left\{t c f(\Pi \bar{\sigma} / D) \mid \bar{\sigma}=\left\langle\sigma_{n} \mid n<\omega\right\rangle, \sigma_{n}^{* *} \leq \sigma_{n}=c f \sigma_{n}<\sigma_{n}^{*}(n<\right.$ $\omega)\} \cap\left[\kappa_{1}, \kappa_{*}\right)$.

(2) There are $\alpha^{*}<\theta$ and a sequence $\left\langle R_{\alpha} \mid \alpha<\alpha^{*}\right\rangle$ with $\bigcup_{\alpha<\alpha^{*}} R_{\alpha}=\operatorname{Reg} \cap \kappa_{*} \backslash \kappa_{1}$ so that $(*)_{2}$ for every $\alpha<\alpha^{*}$ there is $w \leq \gamma^{*}+1$ consisting of at most $n^{*}$ elements such that if $a \in\left[R_{\alpha}\right]^{\aleph_{0}}, \beta \leq \gamma^{*}$ and $\kappa_{*}^{+\beta} \in \operatorname{pcfa}$ then $\beta \in w$.

(3) Let $D$ be a nonprincipal ultrafilter on $\omega$. There is a partition $\left\langle I_{\rho} \mid \rho<\rho^{*}\right\rangle, \rho^{*}<\theta$ of Reg $\cap \kappa_{1} \backslash \kappa_{0}$ into closed open intervals (i.e. of the form $[x, y)$ ) with $\left\langle\min I_{\rho} \mid \rho<\rho^{*}\right\rangle$ strictly increasing such that

$(*)_{3}$ for every sequence $\left\langle\rho_{n} \mid n<\omega\right\rangle$ of ordinals below $\rho^{*}$ with $\lim _{D}\left\langle\min I_{\rho_{n}} \mid n<\omega\right\rangle=$ $\kappa_{1}$

$$
\left\{t c f\left(\prod_{n<\omega} \sigma_{n} / D\right) \mid \sigma_{n} \in I_{\rho_{n}} \text { for } n<\omega\right\} \cap\left[\kappa_{1}, \kappa_{*}\right)
$$

is included in one of $R_{\alpha}$ 's $\left(\alpha<\alpha^{*}\right)$ from a sequence $\left\langle R_{\alpha} \mid \alpha<\alpha^{*}\right\rangle\left(\alpha^{*}<\theta\right)$ satisfying $(*)_{2}$.

Remark 2.1 Part (1) is close to [Sh:g, IX 1.x].

Proof of (2) and (3) from (1). As $\kappa_{*}^{+\gamma^{*}} \leq p p\left(\kappa_{1}\right)$ there are a countable unbounded $a \subseteq \kappa_{1} \cap \operatorname{Reg} \backslash \kappa_{0}$ and an ultrafilter $D_{0}$ on $a$ containing all cobounded subsets of $a$ with $\kappa_{*}^{+\gamma^{*}}=\operatorname{tcf}\left(\Pi a / D_{0}\right)$. Let $a=\left\{\lambda_{n} \mid n<\omega\right\}$ and $D=\left\{A \subseteq \omega \mid\left\{\lambda_{n} \mid n \in A\right\} \in D_{0}\right\}$. Now, by [Sh:g, II], for every regular $\tau \in \kappa_{*}^{+\gamma^{*}} \backslash \kappa_{1}$ we can find $\bar{\sigma}=\left\langle\sigma_{n} \mid n<\omega\right\rangle, \sigma_{n} \in \operatorname{Reg} \cap \kappa_{1} \backslash \kappa_{0}$ $(n<\omega), \lim _{D} \bar{\sigma}=\kappa_{1}$ such that $\tau=\operatorname{tcf}(\Pi \bar{\sigma} / D)$. 
Fix $\chi$ to be a large enough cardinal. Let $M \prec(H(\chi), \epsilon)$ be such that $|M|<\theta,{ }^{\omega} M \subseteq M$, $\left\{\kappa_{0}, \kappa_{1}, \theta, D, \kappa_{*}\right\} \in M$ and $M \cap \theta \in \theta$. There is such $M$ since we assumed (b). Consider the following set $\Phi=\left\{\bar{\sigma}^{*} \mid \bar{\sigma}^{*}=\left\langle\sigma_{n}^{*} \mid n<\omega\right\rangle, \lim _{D} \bar{\sigma}^{*}=\kappa_{1}\right.$ and for every $n<\omega$, $\sigma_{n}^{*} \in M \cap\left[\kappa_{0}^{+}, \kappa_{1}\right)$ is a limit cardinal of cofinality $\left.\geq \theta\right\}$. Clearly, $\Phi \subseteq M$ since ${ }^{\omega} M \subseteq M$. Now, by (1), applied with $D$ defined above for each $\bar{\sigma}^{*} \in \Phi$ there will be $\bar{\sigma}^{* *}$ for which $(*)_{1}$ holds. By elementarity, there is such $\bar{\sigma}^{* *}$ in $M$. Denote it by $\bar{\sigma}^{* *}\left[\bar{\sigma}^{*}\right]$. Define $\left\langle R_{\alpha} \mid \alpha<\alpha^{*}\right\rangle$ to be an enumeration of the set $\left\{R_{D, \bar{\sigma}^{*}, \bar{\sigma}^{* *}\left[\bar{\sigma}^{*}\right]} \mid \bar{\sigma}^{*} \in \Phi\right\} \cup\left\{\left\{t c f\left(\prod_{n<\omega} \sigma_{n} / D\right)\right\} \mid \sigma_{n} \in M \cap \kappa_{1} \cap\right.$ $R e g \backslash \kappa_{0}$ and $\left.\lim _{n<\omega D} \sigma_{n}=\kappa_{1}\right\}$. Then $\alpha^{*}<\theta$ since $|M|<\theta$. Clearly here $(*)_{1}$ implies $(*)_{2}$. So, in order to complete the proof of (2) it remains to show that $\operatorname{Reg} \cap \kappa_{*} \backslash \kappa_{1}=\bigcup_{\alpha<\alpha^{*}} R_{\alpha}$. Let $\tau \in \operatorname{Reg} \cap \kappa_{*} \backslash \kappa_{1}$. Then for some $\bar{\sigma}=\left\langle\sigma_{n} \mid n<\omega\right\rangle, \sigma_{n} \in \operatorname{Reg} \cap \kappa_{1} \backslash \kappa_{0}(n<\omega), \lim _{D} \bar{\sigma}=\kappa_{1}$, $\tau=t c f(\Pi \bar{\sigma} / D)$. Let $A=\left\{n<\omega \mid \sigma_{n} \in M\right\}$.

Case 1. $A \in D$.

Then, w.l. of g. we can assume that $A=\omega$ (just if $\sigma_{n} \notin M$ replace it by $\kappa_{0}^{+}$). But then $\tau$ appears in the second part of the union defining $\left\langle R_{\alpha} \mid \alpha<\alpha^{*}\right\rangle$.

Case 2. $A \notin D$.

Clearly $\kappa_{1} \geq \kappa_{0}^{+\theta}$, since otherwise $\kappa_{1} \cap R e g \subseteq M$ and Case 2 cannot occur. So w.l. of g. we can assume that $A=\emptyset$. Let for $n<\omega, \sigma_{n}^{*}=\min \left(M \cap \kappa_{1} \backslash \sigma_{n}\right)$. Such $\sigma_{n}^{*}$ is well defined since $\kappa_{1} \in M, c f \kappa_{1}=\aleph_{0}$ and hence $\kappa_{1}=\sup \left(\kappa_{1} \cap M\right)$. Also, $\sigma_{n}^{*}$ should be a limit cardinal of cofinality $\geq \theta$ as $M \cap \theta \in \theta$. So $\bar{\sigma}^{*}=\left\langle\sigma_{n}^{*} \mid n<\omega\right\rangle \in \Phi$. Let $\bar{\sigma}^{* *}=\bar{\sigma}^{* *}\left[\bar{\sigma}^{*}\right]$. Now, for every $n<\omega, \kappa_{0}^{+} \leq \sigma_{n}^{* *}<\sigma_{n}^{*}$ and $\sigma_{n}^{* *} \in M$. Hence, $\sigma_{n}^{* *}<\sigma_{n}<\sigma_{n}^{*}$ for every $n<\omega$. Then tcf $(\Pi \bar{\sigma} / D)=\tau \in R_{D, \bar{\sigma}^{*}, \bar{\sigma}^{* *}}$ by $(*)_{1}$ and we are done.

This completes the proof of (2) from (1).

Let us turn now to (3). Here we are given a nonprincipal ultrafilter $D$. Define $M$ and $\left\langle R_{\alpha} \mid \alpha<\alpha^{*}\right\rangle$ as above using this $D$. For every $\nu \in M \cap \kappa_{1} \backslash \kappa_{0}$ a limit cardinal of cofinality $\geq \theta$ denote $\sup (M \cap \nu)$ by $\nu(M)$. Let $\left\langle I_{\rho} \mid \rho<\rho^{*}\right\rangle$ be the increasing enumeration of the following disjoint intervals:

$\left\{\operatorname{Reg} \cap[\nu(M), \nu] \mid \nu \in M \cap \kappa_{1}\right.$ is a limit cardinal of cofinality $\left.\geq \theta\right\} \cup\{\{\nu\} \mid \nu \in M, c f \nu=\nu\}$.

Clearly, $\rho^{*}<\theta$, since $|M|<\theta$. Let us check that $(*)_{3}$ holds. So let $\left\langle\rho_{n} \mid n<\omega\right\rangle$ be a sequence of ordinals below $\rho^{*}$ with $\lim _{D}\left\langle\min I_{\rho_{n}} \mid n<\omega\right\rangle=\kappa_{1}$ and let $\sigma_{n} \in I_{\rho_{n}}$ for $n<\omega$. Consider $\tau=t c f\left(\prod_{n<\omega} \sigma_{n} / D\right)$. Let $A=\left\{n<\omega \mid \sigma_{n} \in M\right\}$. As above we can concentrate on the situation when $A=\emptyset$ (i.e. Case 2). Define $\bar{\sigma}^{*}$ and $\bar{\sigma}^{* *}$ as in Case 2. Then for every $n<\omega, \sigma_{n}^{* *}<\sigma_{n}^{*}$ and $\sigma_{n}^{* *} \in M$. But $\sigma_{n}^{*}=\min \left(M \cap \kappa_{1} \backslash \sigma_{n}\right)$ is a limit cardinal of cofinality $\geq \theta$ in $M$. Let $\tilde{\rho}_{n}$ denote the left side of the interval $I_{\rho_{n}}$. Then $\sigma_{n}^{*}=\tilde{\rho}_{n}$, since $\tilde{\rho}_{n} \in M$ is a limit cardinal of cofinality $\geq \theta$ and $\sigma_{n} \in I_{\rho_{n}}=\left(\sup \left(M \cap \tilde{\rho}_{n}\right), \tilde{\rho}_{n}\right) \cap \operatorname{Reg}$. Also the last equality implies that $\sigma_{n}>\sigma_{n}^{* *}$. Then $\tau=t c f\left(\prod_{n<\omega} \sigma_{n} / D\right) \in R_{D, \bar{\sigma}^{*}, \bar{\sigma}^{* *}}$ and we are done. 
Proof of (1). Suppose otherwise. Let $D$ be a nonprincipal ultrafilter on $\omega$ and $\bar{\sigma}^{*}=$ $\left\langle\sigma_{n}^{*} \mid n<\omega\right\rangle$ a sequence of limit cardinals of cofinality $\geq \theta$ in the interval $\left(\kappa_{0}, \kappa_{1}\right)$ with $\kappa_{1}=\lim _{D} \bar{\sigma}^{*}$ witnessing the failure of (1). We choose by induction on $\xi<\theta$ cardinals $\sigma_{\xi, n}, \tau_{\xi}^{k}, \sigma_{\xi, n}^{k}(n, k<\omega)$ so that

$(\alpha) \kappa_{0}^{+} \leq \sigma_{\xi, n}<\sigma_{n}^{*}$

$(\beta) \xi<\xi^{\prime}$ implies $\sigma_{\xi, n}^{k}<\sigma_{\xi^{\prime}, n}$

$(\gamma) \tau_{\xi}^{k} \in \operatorname{Reg} \cap \kappa_{*} \backslash \kappa_{1}$

$(\delta) \kappa_{*}^{+\gamma} \cap p c f\left(\left\{\tau_{\xi}^{k} \mid k<\omega\right\}\right) \backslash \kappa_{*}$ has at least $n^{*}+1$ members

( $\epsilon) \sigma_{\xi, n}<\sigma_{\xi, n}^{k}<\sigma_{n}^{*}$ and $\sigma_{\xi, n}^{k}$ is regular

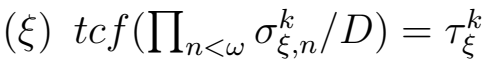

$(\eta) \xi<\xi^{\prime}$ implies that $\sigma_{\xi, n}<\sigma_{\xi^{\prime}, n}$.

In order to carry out the construction we choose first at stage $\xi, \sigma_{\xi, n}$ satisfying $(\alpha),(\beta)$. This is possible, since $\sigma_{n}^{*}$ is a limit cardinal $>\kappa_{0}$ of cofinality $\geq \theta$. Second, as $\left\langle\sigma_{\xi, n} \mid n<\omega\right\rangle$ cannot serve as $\bar{\sigma}^{* *}$ in $(*)_{1}$ by our assumption, there are $\tau_{\xi}^{k} \in R_{D, \bar{\sigma}^{*},\left\langle\sigma_{\xi, n} \mid n<\omega\right\rangle}$ for $k<\omega$ such that $\operatorname{pcf}\left(\left\{\tau_{\xi}^{k} \mid k<\omega\right\}\right) \cap\left(\kappa_{*}, \kappa_{*}^{+\gamma^{*}}\right]$ has at least $n^{*}+1$ members. So clauses $(\gamma),(\delta)$ hold. By the definition of $R_{D, \bar{\sigma}^{*},\left\langle\sigma_{\xi, n} \mid n<\omega\right\rangle}$, we can find for each $k<\omega, \sigma_{\xi, n}^{k} \in \operatorname{Reg} \cap \sigma_{n}^{*} \backslash \sigma_{\xi, n}$ such that $\operatorname{tcf}\left(\prod_{n<\omega} \sigma_{\xi, n}^{k}, D\right)=\tau_{\xi}^{k}$. So clauses $(\epsilon)$ and $(\xi)$ hold. The clause $(\eta)$ is implied by the previous ones. So, we have finished the inductive construction.

Now, for every $n<\omega$, as $\left\langle\sigma_{\xi, n} \mid \xi<\theta\right\rangle$ is strictly increasing, its limit $\sigma_{n}=\bigcup_{\xi<\theta} \sigma_{\xi, n}$ is a singular cardinal of cofinality $\theta$. Also, clearly, $\sigma_{n} \in\left[\kappa_{0}^{+}, \kappa_{1}\right)$. Hence, by the assumption $(d)$ of the theorem, $p p \sigma_{n} \leq \sigma_{n}^{+n^{*}}$. For $\ell=1, \ldots, n^{*}$ let $\lambda_{\ell}=t c f\left(\prod_{n<\omega} \sigma_{n}^{+\ell} / D\right)$. Set $w^{*}=\left\{\alpha \leq \gamma^{*} \mid \kappa_{*}^{+\alpha}=\lambda_{\ell}\right.$ for some $\left.\ell, 1 \leq \ell \leq n^{*}\right\}$. Then $w^{*}$ is a set of $\leq n^{*}$ ordinals below $\gamma^{*}+1$. Let $a_{n}=\left\{\sigma_{\xi, n}^{k} \mid k<\omega, \xi<\theta\right\}$ and $a=\bigcup_{n<\omega} a_{n} \cup\left\{\sigma_{n}^{+\ell} \mid n<\omega, 1 \leq \ell \leq n^{*}\right\}$. So, $a$ is a set of $\leq \theta<\kappa_{0}<\min a$ regular cardinals. By [Sh:g, VIII §2] or [Sh:506, §2] $a$ has a generating sequence $\left\langle b_{\tau} \mid \tau \in p c f a\right\rangle$. For each $\xi<\theta$ we can find a successor ordinal $\gamma_{\xi} \leq \gamma^{*}$ so that $\kappa_{*}^{+\gamma_{\xi}} \in \operatorname{pcf}\left(\left\{\tau_{\xi}^{k} \mid k<\omega\right\}\right) \backslash\left\{\lambda_{\ell} \mid 1 \leq \ell \leq n^{*}\right\}$. So, for some successor ordinal $\gamma^{* *} \leq \gamma^{*}$ there is an unbounded in $\theta$ set $Y$ consisting of $\xi$ 's such that $\xi<\theta$ and $\gamma_{\xi}=\gamma^{* *}$. Clearly, $\lambda_{\ell} \in p c f a$ for $\ell=1, \ldots, n^{*}$ and $\kappa_{*}^{+\gamma^{* *}} \in p c f a$. Then w.l. of $g$. we can assume that $b_{\kappa_{*}^{+\gamma^{* *}}}$ is disjoint to each $b_{\lambda_{\ell}}$ for $\ell=1, \ldots, n^{*}$. Set $A=\left\{n<\omega \mid b_{\kappa_{*}^{+\gamma^{* *}}} \cap \sigma_{n}\right.$ is unbounded in $\left.\sigma_{n}\right\}$.

Claim 2.2 $A \in D$. 
Proof. If this does not hold, then there is $\xi(*)<\theta$ such that for every $n \in \omega \backslash A b_{\kappa_{*}^{+\gamma^{* *}}} \cap$ $\left[\sigma_{\xi(*)}, \sigma_{n}\right)=\emptyset$. W.l. of g. $\xi(*) \in Y$. Also, $n \in \omega \backslash A$ implies that $\left\{\sigma_{\xi(*), n}^{k} \mid k<\omega\right\} \cap b_{\kappa_{*}^{+\gamma^{* *}}}=\emptyset$, since for every $k<\omega, \sigma_{\xi(*)}<\sigma_{\xi(*), n}^{k}<\sigma_{n}$.

Hence $\left\{\sigma_{\xi(*), n}^{k} \mid k<\omega, n \in \omega \backslash A\right\}$ is disjoint to $b_{\kappa_{*}^{\gamma^{* *}}}$. Now, each $\tau_{\xi(*)}^{k} \in \operatorname{pcf}\left(\left\{\sigma_{\xi(*), n}^{k^{\prime}} \mid k^{\prime}<\right.\right.$ $\omega, n \in \omega \backslash A\})$. Here we use the assumption that $A \notin D$ and so $\omega \backslash A \in D$.

But $\kappa_{*}^{+\gamma^{* *}} \in \operatorname{pcf}\left(\left\{\tau_{\xi(*)}^{k} \mid k<\omega\right\}\right)$. Hence $\kappa_{*}^{+\gamma^{* *}} \in \operatorname{pcf}\left(\left\{\sigma_{\xi(*), n}^{k} \mid k<\omega, n \in \omega \backslash A\right\}\right) \subseteq$ $p c f\left(a \backslash b_{\kappa_{*}^{+\gamma^{* *}}}\right)$, which is impossible by the choice of generators.

$\square$ of the claim.

Let $n \in A$. Then $b_{\kappa_{*}^{+\gamma^{* *}} \cap} \cap \sigma_{n}$ is unbounded in $\sigma_{n}$. Hence $p c f\left(b_{\kappa_{*}^{+\gamma^{* *}}} \cap \sigma_{n}\right) \backslash \sigma_{n} \neq \emptyset$. But $p p\left(\sigma_{n}\right) \leq \sigma_{n}^{+n^{*}}$, hence for some $\ell(n) \in\left\{1, \ldots, n^{*}\right\}$ we have $\sigma_{n}^{+\ell(n)} \in \operatorname{pcf}\left(b_{\kappa_{*}^{+*^{* *}}} \cap \sigma_{n}\right) \subseteq$ $p c f\left(b_{\kappa_{*}^{+} *^{* *}}\right)$. Then for some $\ell(*) \in\left\{1, \ldots, n^{*}\right\}$ the set $A^{*}=\{n \in A \mid \ell(n)=\ell(*)\}$ belongs to $D$. So, $\lambda_{\ell(*)} \in \operatorname{pcf}\left(\left\{\sigma_{n}^{+\ell(*)} \mid n \in A^{*}\right\}\right) \subseteq p c f\left(b_{\kappa_{*}^{+\gamma^{* *}}}\right)$. But $b_{\kappa_{*}^{+\gamma^{* *}}} \cap b_{\lambda_{\ell(*)}}=\emptyset$. Contradiction.

Using (3) of Theorem 2 we can give another proof of Theorem 1.

\subsection{Second proof of Theorem 1}

W.l. of g. $c f \kappa_{*}=\left(2^{\aleph_{0}}\right)^{+}$. Let $\theta=\left(2^{\aleph_{0}}\right)^{+}$and $\kappa_{0}=\theta^{+}$. Assume also w.l. of g. that $D$ is a nonprincipal ultrafilter on $\omega$. For every $f: \omega \rightarrow \operatorname{Reg} \cap \kappa_{1} \backslash \kappa_{0}$ we define $g_{f}: \omega \rightarrow \rho^{*}<\theta$ as follows:

$$
g_{f}(n)=\rho \quad \text { iff } \quad f(n) \in I_{\rho} .
$$

Then, $f_{1} \geq_{D} f_{2}$ will imply $g_{f_{1}} \geq_{D} g_{f_{2}}$ since the sequence $\left\langle\min I_{\rho} \mid \rho<\rho^{*}\right\rangle$ is strictly increasing. Consider $\left\langle f_{\lambda_{\alpha}} \mid \alpha<\theta\right\rangle$ of (d) of Theorem 1. This is a strictly increasing sequence modulo $D$. Now, the total number of $g_{f}$ 's is $\left(\rho^{*}\right)^{\aleph_{0}} \leq\left(2^{\aleph_{0}}\right)^{\aleph_{0}}=2^{\aleph_{0}}$. Hence there are $g^{*}: \omega \rightarrow \rho^{*}$ and $\alpha^{*}<\theta$ such that for every $\alpha, \theta>\alpha \geq \alpha^{*}$, every $f: \omega \rightarrow \operatorname{Reg} \cap \kappa_{1} \backslash \kappa_{0}$ such that $f_{\lambda_{\alpha}} \leq_{D} f<_{D} f_{\lambda_{\alpha+1}}$

$$
f(n) \in I_{g^{*}(n)}, \text { for almost each } n<\omega \bmod D .
$$

Apply $(*)_{3}$ to $\left\langle g^{*}(n) \mid n<\omega\right\rangle$ with $\gamma^{*}=2$. Then for some $\ell^{*} \in\{1,2\}$ the following holds: if $a \in\left[\left\{t c f\left(\prod_{n<\omega} \sigma_{n} / D\right) \mid \sigma_{n} \in I_{g^{*}(n)} \text { for } n<\omega\right\} \cap\left[\kappa_{1}, \kappa^{*}\right)\right]^{\aleph_{0}}$ then $\kappa_{*}^{+\ell^{*}} \notin p c f a$. Let $\alpha$, $\theta>\alpha \geq \alpha^{*}$. Pick $\kappa_{\alpha}, \lambda_{\alpha}<\kappa_{\alpha}<\lambda_{\alpha+1}, c f \kappa_{\alpha}=\aleph_{0}$ and $p p \kappa_{\alpha} \geq \kappa_{*}^{++}$(by (c) of Theorem 1 we can assume w.l. of g. that it exists). Then, by [Sh-g], there are $\tau_{\alpha, n} \in \operatorname{Reg} \cap \kappa_{\alpha} \backslash \lambda_{\alpha}^{++}$ $(n<\omega)$ and a filter $D_{\alpha}$ on $\omega$ containing all cofinite sets such that $\kappa_{*}^{+\ell^{*}}=t c f\left(\prod_{n<\omega} \tau_{\alpha, n} / D_{\alpha}\right)$. Consider $\left\langle f_{\tau_{\alpha, n}}(m) \mid m<\omega\right\rangle$ for every $n<\omega$. It is a sequence of regular cardinals such that $\tau_{\alpha, n}=\operatorname{tcf}\left(\prod_{m<\omega} f_{\tau_{\alpha, n}}(m) / D\right)$ and $f_{\lambda_{\alpha}}<_{D} f_{\tau_{\alpha, n}}<_{D} f_{\lambda_{\alpha+1}}$. Then for almost every $m<\omega$ 
$(\bmod D) f_{\tau_{\alpha, n}}(m) \in I_{g^{*}(m)}$. Hence $\tau_{\alpha, n} \in\left\{t c f\left(\prod_{m<\omega} \sigma_{m} / D\right) \mid \sigma_{m} \in I_{g^{*}(m)}, m<\omega\right\}$ for every $n<\omega$. Take $a=\left\{\tau_{\alpha, n} \mid n<\omega\right\}$. Then $k_{*}^{+\ell^{*}} \notin p c f a$, but $\kappa_{*}^{+\ell^{*}}=t c f\left(\prod_{n<\omega} \tau_{\alpha, n} / D_{n}\right)$. Contradiction.

The following is parallel to 1.6.

\section{Theorem 2.4 Suppose that}

(a) $\kappa_{0}<\kappa_{1}<\kappa_{*}$

(b) $\theta_{1}, \theta_{2}<\kappa_{0}$ are such that cf $\theta_{1}>\aleph_{0}, \theta_{2}=\theta_{1}^{+3}$ or $\theta_{2}$ is regular $\geq \theta_{1}^{+3}$ and for every $\alpha<\theta_{2} \quad c f\left([\alpha]^{<\theta_{1}}, \supseteq\right)<\theta_{2}$

(c) $c f \kappa_{1}=\aleph_{0}$ and $p p \kappa_{1} \geq \kappa_{*}^{+\theta_{2}}$

(d) $\theta_{3}$ is regular cardinal between $\theta_{2}$ and $\kappa_{0}$

(e) $\theta_{4}$ is cardinal between $\theta_{3}$ and $\kappa_{0}$ of cofinality $\geq \theta_{3}$

(f) $\theta_{5} \in\left[\theta_{4}, \kappa_{0}\right)$ is a cardinal such that $\operatorname{cf}\left(\left[\theta_{5}\right]^{\leq \aleph_{0}}, \subseteq\right)=\theta_{5}$

(g) $D$ is an $\aleph_{1}$-complete filter on $\theta_{4}+1$

(Notice that we allow $D$ to be principal. For example, generated by $\left\{\theta_{4}\right\}$ ).

(h) if $\left\langle\mu_{\alpha} \mid \alpha \leq \theta_{4}\right\rangle$ is a strictly increasing continuous sequence of singular cardinals between $\kappa_{0}$ and $\kappa_{1}$, then

$$
\left\{\alpha \leq \theta_{4} \mid \alpha \text { limit, cf } \mu_{\alpha} \geq \theta_{4} \text { and } p p\left(\mu_{\alpha}\right)<\mu_{\alpha}^{+\theta_{1}}\right\} \in D
$$

(Thus, if $\left\{\theta_{4}\right\} \in D$ then the condition means $p p(\mu)<\mu^{+\theta_{1}}$ for every limit cardinal $\mu \in\left(\kappa_{0}, \kappa_{1}\right)$ of cofinality $\theta_{4}$.)

Then

(1) For every sequence $\bar{\sigma}^{*}=\left\langle\sigma_{n}^{*} \mid n<\omega\right\rangle$ of limit cardinals of cofinality $\geq \theta_{4}$ between $\kappa_{0}^{+}$ and $\kappa_{1}$ there are $\beta<\theta_{2}$ and a sequence $\bar{\sigma}^{* *}=\left\langle\sigma_{n}^{* *} \mid n<\omega\right\rangle, \kappa_{0}^{+} \leq \sigma_{n}^{* *}<\sigma_{n}^{*}(n<\omega)$ such that

$(\tilde{*})_{1}$ if $a \in\left[R_{\bar{\sigma}^{*}, \bar{\sigma}^{* *}}\right]^{\aleph_{0}}$ then pcf $(a) \cap\left[\kappa_{*}^{+\beta}, \kappa_{*}^{+\theta_{2}}\right)=\emptyset$, where $R_{\bar{\sigma}^{*}, \bar{\sigma}^{* *}}=\left\{\tau \in\left(\kappa_{0}^{+}, \kappa_{1}\right) \mid\right.$ there is a sequence $\left\langle\sigma_{n} \mid n<\omega\right\rangle$, with $\sigma_{n} \in \operatorname{Reg} \cap\left[\sigma_{n}^{* *}, \sigma_{n}^{*}\right)$ such that $\tau \in \operatorname{pcf}\left\{\sigma_{n} \mid n<\right.$ $\omega\}\}$. 
(2) There are $\alpha^{*} \leq \theta_{5}$ and a sequence $\left\langle R_{\alpha} \mid \alpha<\alpha^{*}\right\rangle$ with $\bigcup_{\alpha<\alpha^{*}} R_{\alpha}=R e g \cap \kappa_{*} \backslash \kappa_{1}$ so that $(\tilde{*})_{2}$ for every $\alpha<\alpha^{*}$ there is $\beta<\theta_{2}$ such that for every $a \in\left[R_{\alpha}\right]^{\aleph_{0}}$ we have $\operatorname{pcf}(a) \cap$ $\left[\kappa_{*}^{+\beta}, \kappa_{*}^{+\theta_{2}}\right)=\emptyset$.

(3) There are $\rho^{*}<\theta_{5}^{+}$and a partition $\left\langle I_{\rho} \mid \rho<\rho^{*}\right\rangle$ of Reg $\cap \kappa_{1} \backslash \kappa_{0}$ into closed open intervals (i.e. of the form $[x, y)$ ) with $\left\langle\min I_{\rho} \mid \rho<\rho^{*}\right\rangle$ strictly increasing such that

$(\tilde{*})_{3}$ for every sequence of ordinals $\left\langle\rho_{n} \mid n<\omega\right\rangle$ below $\rho^{*}$ there is $\beta<\theta_{2}$ such that for every $a \in\left[\left\{t c f\left(\prod_{n<\omega} \sigma_{n} / \widetilde{D}\right) \mid \sigma_{n} \in I_{\rho_{n}}\right.\right.$ for $n<\omega, \widetilde{D}$ is a nonprincipal ultrafilter on $\omega$ with $\left.\left.\lim _{n<\omega} D\left(\min I_{\rho_{n}}\right)=\kappa_{1}\right\}\right]^{\aleph_{0}}$

$$
p c f(a) \cap\left[\kappa_{*}^{+\beta}, \kappa_{*}^{+\theta_{2}}\right)=\emptyset .
$$

\section{Proof of (2) and (3) from (1)}

Let $\chi$ be a large enough cardinal. Pick $M \prec(H(\chi), \epsilon)$ so that $|M|=\theta_{5}, \kappa_{0}, \kappa_{1}, \theta_{5} \in M$, $M \cap \theta_{5}^{+} \in \theta_{5}^{+}$and $\left(\forall X \in[M]^{\aleph_{0}}\right)(\exists Y \in M)\left(X \subseteq Y \wedge|Y|=\aleph_{0}\right)$.

This is possible since by (f) $c f\left(\left[\theta_{5}\right]^{\leq \aleph_{0}}, \subseteq\right)=\theta_{5}$. Define the set $\Phi$ now to be $\left\{\bar{\sigma}^{*} \in M \mid\right.$ $\bar{\sigma}^{*}=\left\langle\sigma_{n}^{*} \mid n<\omega\right\rangle$ is a sequence of limit cardinals between $\kappa_{0}$ and $\kappa_{1}$ with $c f \sigma_{n}^{*} \geq \theta_{4}$ $(n<\omega)\}$.

For each $\bar{\sigma}^{*} \in \Phi$ we choose $\bar{\sigma}^{* *}=\bar{\sigma}^{* *}\left[\bar{\sigma}^{*}\right]$ in $M$ satisfying $(\widetilde{*})_{1}$. Define $\left\langle R_{\alpha} \mid \alpha<\alpha^{*}\right\rangle$ to be an enumeration of the set $\left\{R_{\bar{\sigma}^{*}, \bar{\sigma}^{* *}\left[\bar{\sigma}^{*}\right]} \mid \bar{\sigma}^{*} \in \Phi\right\} \cup\left\{p c f\left(\left\{\sigma_{n} \mid n<\omega\right\}\right) \mid\left\langle\sigma_{n} \mid n<\omega\right\rangle \in \Phi\right.$ and for every $\left.n<\omega \quad c f \sigma_{n}=\sigma_{n}\right\}$.

Now we proceed as in Theorem 2 .

Proof of (1). Assume toward contradiction that for some $\bar{\sigma}^{*}$ there is no $\bar{\sigma}^{* *}$ satisfying (1). We choose by induction on $\xi<\theta_{4}$ cardinals $\sigma_{\xi, n}, \tau_{\xi}^{i, k}, \sigma_{\xi, n}^{i, k}\left(k, n<\omega, i<\theta_{2}\right)$ such that

$(\alpha) \kappa_{0}^{+} \leq \sigma_{\xi, n}<\sigma_{n}^{*}$

$(\beta) \xi<\xi^{\prime}$ implies that $\sigma_{\xi, n}^{i}<\sigma_{\xi^{\prime}, n}$

$(\gamma) \tau_{\xi}^{i, k} \in \operatorname{Reg} \cap \kappa_{*} \backslash \kappa_{1}$

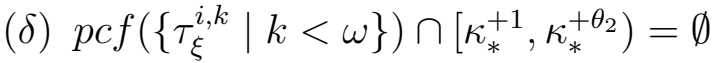

(є) $\tau_{\xi}^{i, k} \in \operatorname{pcf}\left(\left\{\sigma_{\xi, n}^{i, k} \mid n<\omega\right\}\right)$

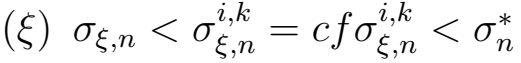

( $\eta)\left\langle\sigma_{\xi, n} \mid \xi<\theta_{4}\right\rangle$ is an increasing continuous sequence of singular cardinals. 
The verification that such a construction is possible is as in the proof of (1) of Theorem 2.

Let $\sigma_{n}=\sigma_{n, \theta_{4}}=\bigcup_{\xi<\theta_{4}} \sigma_{\xi, n}$ for each $n<\omega$. Applying the condition (h) of the statement of the theorem to $\left\langle\sigma_{\xi, n} \mid \xi \leq \theta_{4}\right\rangle$ we find for every $n<\omega$ a set $Y_{n} \in D$ such that $\xi \in Y_{n}$ implies that $p p\left(\sigma_{\xi, n}\right)<\sigma_{\xi, n}^{+\theta_{1}}$. By $\aleph_{1}$-completeness of $D$, the set $Y=\bigcap_{n<\omega} Y_{n} \in D$. Choose some $\delta^{*} \in Y$. Let $p p\left(\sigma_{\delta^{*}, n}\right)=\left(\sigma_{\delta^{*}, n}\right)^{+\beta_{n}}$ for some $\beta_{n}<\theta_{1}(n<\omega)$.

Consider sets $a_{n}=\left\{\sigma_{\xi, n}^{i, k} \mid \xi<\delta^{*}, i<\theta_{2}, k<\omega\right\}$ and $a=\left(\bigcup_{n<\omega} a_{n}\right) \cup a^{*}$, where $a^{*}=\left\{\left(\sigma_{\delta^{*}, n}\right)^{+\beta} \mid n<\omega, \beta \leq \beta_{n}\right.$ is a successor ordinal $\}$. Then $a$ is a set of regular cardinals of cardinality $\leq \theta_{4}+\theta_{2}<\kappa_{0}<\min a$. Let $\left\langle b_{\tau} \mid \tau \in p c f a\right\rangle$ be a generating sequence. As each $\beta_{n}<\theta_{1}$ and $c f \theta_{1}>\aleph_{0},\left|a^{*}\right|<\theta_{1}$. By [Sh:g, IX] or [Sh:g, E12, 4.18(b)] $c=p c f\left(a^{*}\right) \cap\left[\kappa_{*}, \kappa_{*}^{+\theta_{2}}\right)$ is bounded in $\kappa_{*}^{+\theta_{2}}$, since $\theta_{2} \geq \theta_{1}^{+3} \geq\left|a^{*}\right|^{+4}$. Also pcf $(c)=c$. For each $\xi<\delta^{*}$ for some $i(\xi)$ we have $p c f\left(\left\{\tau_{\xi}^{i(\xi), k} \mid k<\omega\right\}\right) \cap\left[\kappa_{*}, \kappa_{*}^{+\theta_{2}}\right)$ is not bounded by $\sup c$. So, choose $\kappa_{*}^{+\rho(\xi)} \in \operatorname{pcf}\left(\left\{\tau_{\xi}^{i(\xi), k} \mid k<\omega\right\}\right) \cap\left[\kappa_{*}, \kappa_{*}^{+\theta_{2}}\right) \backslash \sup c$. Clearly, $\rho(\xi)<\theta_{2}$ is a successor ordinal. As, $\theta_{2}<\theta_{3}=\operatorname{cf} \theta_{3}$, and $\delta^{*} \in Y$ implies either $\left(c f \delta^{*}=\theta_{3}\right)$ or $\left(\delta^{*}=\theta_{4}\right.$ and then also cf $\left.\delta_{1}^{*} \geq \theta_{3}\right)$, necessary, for some $\rho^{*}<\theta_{2}$ the set $Z=\left\{\xi<\delta^{*} \mid \rho(\xi)=\rho^{*}\right\}$ is unbounded in $\delta^{*}$. Let $J_{n}=J_{a_{n}}^{b d}$. So $J_{n}$ is an ideal on $a_{n}$ and, clearly, for every $c_{n} \in J_{n}(n<\omega)$ we have $\kappa_{*}^{+\rho^{*}} \in \operatorname{pcf}\left(\bigcup_{n<\omega}\left(a_{n} \backslash c_{n}\right)\right)$.

By $p c f$ theory (see [Sh:g, VIII, 1.5] or [Sh:g, E12]) there are finite sets $e_{n} \subseteq \cap\left\{p c f\left(a_{n} \backslash c_{n}\right) \mid\right.$ $\left.c_{n} \in J_{n}\right\}(n<\omega)$ such that $\kappa_{*}^{+\rho^{*}} \in \operatorname{pcf}\left(\bigcup_{n<\omega} e_{n}\right)$. But $\cap\left\{p c f\left(a_{n} \backslash c_{n}\right) \mid c_{n} \in J_{n}\right\} \subseteq\left\{\sigma_{\delta^{*}, n}^{+\beta} \mid\right.$ $\beta<\beta_{n}$ is a successor ordinal $\}$ for every $n<\omega$. So $\bigcup_{n<\omega} e_{n} \subseteq \cup\left\{\sigma_{\delta^{*}, n}^{+\beta} \mid \beta<\beta_{n}\right.$ is a successor ordinal and $n<\omega\}=a^{*}$. Hence, $\kappa_{*}^{+\rho^{*}} \in \operatorname{pcf}\left(a^{*}\right)$. But then $\kappa_{*}^{+\rho^{*}} \in \operatorname{pcf}\left(a^{*}\right) \cap\left[\kappa_{*}, \kappa_{*}^{+\theta_{2}}\right)=c$, which is impossible by the choice of $\rho^{*}$. Contradiction.

Let us conclude with a question which is most natural taking into account the results above.

Question. Is the following situation possible:

(a) $\kappa_{1}<\kappa_{*}, c f \kappa_{1}=\aleph_{0}, c f \kappa_{*}=\aleph_{1}$

(b) for every singular $\mu<\kappa_{1}, p p \mu=\mu^{+}$(or if one likes only for $\mu$ 's of countable cofinality)

(c) $\kappa_{*}=\sup \left\{\mu \mid \mu<\kappa_{*}, c f \mu=\aleph_{0}\right.$ and $\left.p p \mu=\kappa_{*}^{+}\right\}$

(d) the same as (d) of Theorem 1 or even add $(*)$ of the proof of Theorem 1.

\section{References}

[Gi] M. Gitik, Blowing power of a singular cardinal-wider gaps. 
[Gi-Ma] M. Gitik and M. Magidor, Extender based forcings, JSL 59(2) (1994), 450-460.

[Gi-Sh] M. Gitik and S. Shelah, On certain indestructibiltiy of strong cardinals and a question of Hainal, Arch. Math. Logic 28 (1989), 35-42.

[Sh:400A] S. Shelah, Cardinal arithmetic for skeptics, Bulletin of the AMS 26(2) (1992), 197-210.

[Sh:g] S. Shelah, Cardinal arithmetic, Oxford Science Publ., Oxford Logic Guides: 29 (1994). 